\title{
A Novel Spectrum Allocation Algorithm based on Graph Coloring
}

\author{
Wenzhun Huang $^{\mathrm{a}^{*}}$, Xinxin Xie ${ }^{\mathrm{b}}$ and Hui Zhang ${ }^{\mathrm{c}}$ \\ ${ }^{1}$ Department of Electronic Information Engineering, Xijing University, Xi'an 710123, China \\ ahuangwenzhun@xijing.edu.cn, b346148500@qq.com, 'czhang_hui@xijing.edu.cn
}

\begin{abstract}
Keywords: Spectrum Allocation, Communication, Graph Coloring, Topology Optimization.
Abstract. In this paper, we conduct research on the novel spectrum allocation algorithm based on the graph coloring. Today, with the constant development of wireless communication technology, people demand for it is growing. The current communication resources are becoming increasingly nervous, the lack of spectrum resources become a key problem faced in the process of wireless applications. In cognitive radio network bandwidth, the number and location of available channel are changing with time, so the spectrum allocation method in the traditional network does not fully apply. To achieve the full spectrum allocation is limited by many policy standard, this paper studies the coloring of graph theory is a classical optimization theory, at the same time in the meet restrictions also consider the allocation of resources of the network. We review the state-of-the-art related theories to form better organization of the system that enhances the overall performance.
\end{abstract}

\section{Introduction}

Wireless sensor network is a distributed sensing information system, composed of spatial distribution and collaborate more nodes. These nodes have very limited computation, the wireless communication and storage space and energy. With the deepening of the research, scalability and maintainability of the wireless sensor network has become an important target of its application promotion. The wireless sensor network has the characteristic such as limited resources, diversity of hardware and application makes the sensor network application development and deployment is more difficult. This requires developing the middleware architecture, providing appropriate abstraction layer to hide the operating systems and hardware technical details, under the condition of the limited energy resources to deploy applications to the wireless sensor network so quickly [1-2].

In the general wireless transmission of wireless sensor network system design, most of the power consumption of wireless communication system, transmission distance, wireless transmission rate, data read back rate requirements, this needs in the wireless sensor network and wireless transmission system in the hardware design that want to choose the indicators and can meet the corresponding requirements of the device. As for the corresponding applications on the spectrum allocation, it can be generally summarized as the following principles. (1) Performance evaluation module. Performance evaluation module is located in network side, maintained the past cycle spectrum allocation decision history information, and is responsible for collecting basic idle spectrum use feedback report from the cognitive user terminal, thereby supporting spectrum allocation decisions modules, to improve the performance of the dynamic spectrum allocation decisions. (2) Spectrum allocation decision module. Spectrum allocation decision module is located in network side. Its main function is to collect all the idle spectrum detection and application of the cognitive users request information, and according to decision of past cycle history that make a new round of spectrum allocation decisions. (3) Spectrum sequence selection module is located in the cognitive user terminal Its main function is based on the current application of the quality of service requirements to calculate the cognitive user preference for the idle spectrum resource, and the corresponding information on the spectrum allocation decision as module on to the general and target network [3-4].

In recent years, in the field of cognitive radio spectrum allocation of resources to get the in-depth research and extensive attention, its main models are auction bidding model, model of game theory, and theory of graph coloring model and other model and the dynamic spectrum allocation in cognitive radio technology can rely on artificial intelligence wireless communication environment perception, dynamic detection and effective use of the idle spectrum, adaptive adjustment of system 
parameters, significantly reduce spectrum and bandwidth limitations of wireless technology development. This technology is predicted for the future of the most popular wireless technology. In the following figure one, we demonstrate the contemporary spectrum allocation pattern and feature.

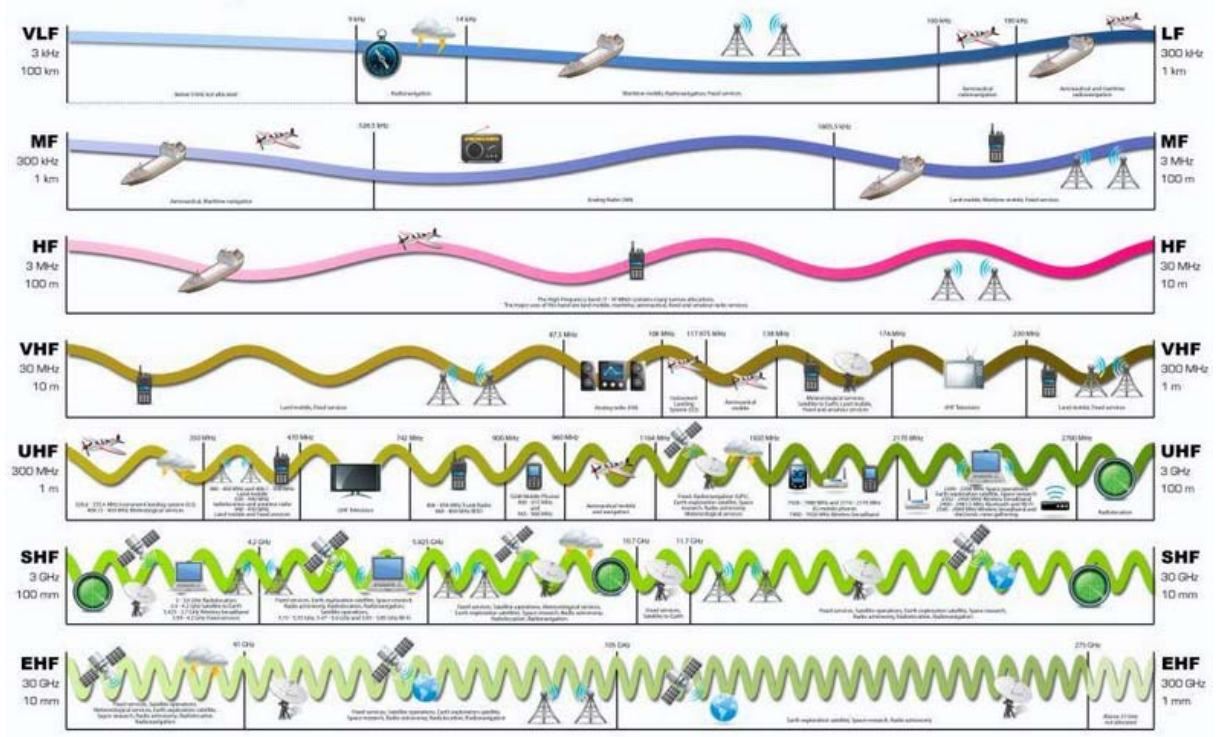

Figure 1. The Contemporary Spectrum Allocation Pattern and Feature

In this paper, we conduct research on the novel spectrum allocation algorithm based on the graph coloring. The existing dynamic spectrum allocation based on the basic graph theory are analyzed and summarized, but each algorithm has some shortcomings, need to do further improvement, especially on operation costs. How to assign spectrum effective cognitive users, they can do at the same time maximize primary channel utilization combined with interference minimizing and allocation equity consideration, cognitive radio and the dynamic spectrum allocation is the future research focus and the direction. In the later parts, we will introduce our methodology in detail.

\section{The Proposed Algorithm}

The General Feature of the Wireless Sensor Network. In general, typical wireless sensor network consists of sensor nodes, sink node, the Internet and the user interface, etc. Through the planes had been planting seed, the artificial place, in the interior of the perception object or surrounding a large number of sensor nodes deployed. Composition between nodes by the self-organizing wireless sensor network, and general perception, acquisition and processing in collaborative manner the information specified in the network coverage area, achieve any position within the target area for collection of the real-time information with the purpose of processing and analysis [5].

The development of wireless sensor network is changing, but there are still a big gap from mature application demand, research of the wireless sensor network topology control is still not mature, not system, cannot meet the requirements of practical application, so it remains to be improved in many ways. In summary primary techniques involved could be summarized as follows. (1) In determining the wireless sensor network technology for application system design, the first problem is to use what network model. Whether have infrastructure support, if there is a mobile terminal, report frequency and delay directly determines the networking application demand. (2) Media access control and link control to solve the wireless network in the prevalent problems of conflict and lost, according to the state of the network data flow control near nodes, and all the nodes in the network channel access method and sequence, to achieve efficient use of network capacity, reduce energy consumption. (3) Networking mode determines the overall topology of network, but in order to realize the network of low energy consumption operation that also need to fine-grained control time varying rule of the node connections. The topology of the network topology in the limited switch between space control by controlling node sends power to change the connected area that make the network presents different connecting forms, thus control the effect of energy consumption, improve network capacity as logic control is through the neighbor table will not be excluded to form a more stable and robust topology. 
The Spectrum Allocation Principles. Of cognitive radio technology is regarded as the most direct means to solve these problems. Based on the cognitive radio technology of the node has the capability of environmental perception and general transmission parameters configuration. Such nodes by cyclic spectrum efficiency of environment perception information and intelligent learning, opportunistic access to authorized users of the free business transmission spectrum resources, so as to realize the dynamic use of spectrum resources. With aid of cognitive radio technology, free spectrum allocated dynamically. Effective spectrum allocation is the key to the dynamic spectrum access technology difficult problem that the existing research mainly through channel load dynamic spectrum allocation optimization. However, further research results show that the simple optimization according to the load spectrum allocation is inefficient, in the actual network environment, may be counter-productive. Near-far effect problem is common in wireless access networks, far from the access point users of low signal noise ratio and the transmission rate is lower than near the AP user, under the mechanism of the random access takes up channel for a long time, from transmission time close to the user. Therefore, near-far effect is one of the causes of the AP load increase. The spectrum allocation algorithm based on load do not distinguish between the reasons of the load to the near-far effect caused by the load increase, the wider channel does not necessarily can improve systematic [6-7].

Perception is the main task of cognitive radio spectrum environment it embodies the most notable features of cognitive radio: sensing and analysis of the spectrum of a particular area, find out the suitable spectrum holes. Generally speaking, the cognitive radio technology that can be divided into perception based on transmitter detection, cooperation based on the interference test. Cognitive radio is through the detection spectrum, to communicate with the empty space. So the authorized users to communicate in the first condition is to detect the authorized user idle spectrum, as if the first user's signal within a certain band is present, cognitive radio should have the ability to detect this signal. We could establish a model of a hypothesis test as the formula one.

$$
x(t)=\left\{\begin{array}{c}
s(t)+n(t) H_{1} \\
n(t) H_{0}
\end{array}\right.
$$

Matched filter detection is one of the biggest advantages on the output side can maximize the signal-to-noise ratio of the signal, and in a short time can obtain higher processing gain. It requires, first of all, know a priori information of authorized users signal if the information is not accurate, the test results will be affected by a lot of. It is a kind of coherent detection, thus to phase synchronization requirement is very high, computation quantity is big, and all kinds of the authorized users, cognitive radio should have a dedicated receiver, this increases the resource consumption and complexity of the system. Correspondingly, we define the related parameters as the following formulas.

$$
\begin{aligned}
& P_{f}=Q\left((\lambda-\varepsilon) / \sqrt{\varepsilon \sigma^{2}}\right) \\
& P_{d}=Q\left((\lambda) / \sqrt{\varepsilon \sigma^{2}}\right)
\end{aligned}
$$

Modulation of the primary user signal is usually carrier, pulse sequence and the frequency hopping sequences or cyclic prefix and so on, all of these can make the inner periodic signal. The modulation signal can be showed smooth circulation features, because they all mean and autocorrelation function of the present a cyclical, same as the signal cycle and cycle.

The Graph Coloring based Allocation Algorithm. The mathematical model of basic spectrum allocation is built on a certain constraint conditions. In the study of the cognitive radio system, can be composed of cognitive user network topology abstraction into a figure. Cognitive user's spectrum can be equivalent to graph vertex coloring problem. Each vertex in the figure represents a cognitive user, colors or basic channel frequency, each side said the conflict between the corresponding vertex or the interference, which cannot be used at the same time the same spectrum. At the same time, each vertex is associated with a collection, the collection of elements in the said the vertex location area can use the spectrum. Due to each vertex of the geographical position is different, so general different vertex associated elements in the collection may be different [8]. 


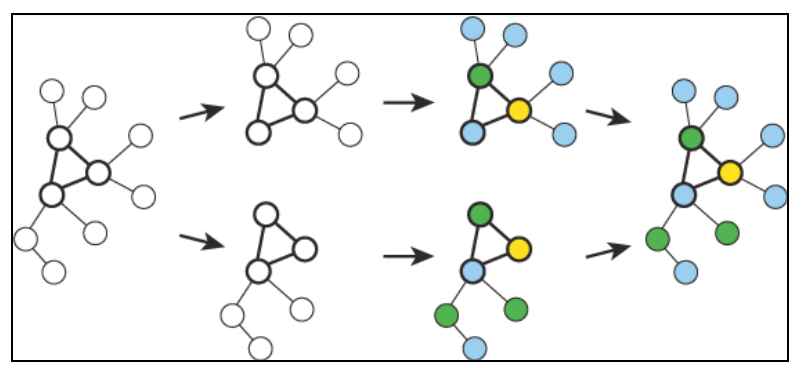

Figure 2. The Illustration of the Graph Coloring Concept

The purpose of spectrum allocation in cognitive radio network is on the premise of without causing interference authorized users, as far as possible improve spectrum utilization system and guarantee the fairness between users. Due to changes in real time and the working state of authorized user time domain, frequency domain and spatial domain existence difference, so in the process of spectrum allocation, it is difficult to achieve the higher cognitive users benefit. According to the graph coloring model and in the open spectrum in wireless network list based on the chromatic spectrum allocation algorithm is proposed, the goal is to achieve maximum spectrum efficiency as the follows.

$$
\arg \max \sum_{i=1}^{N} \sum_{k=1}^{K} S_{i k} \quad \text { s.t. }\left\{\begin{array}{c}
s_{i k} \leq l_{i k} \\
s_{i k} s_{j k} e_{i j}=0 \\
s_{i k}=0 \wedge 1
\end{array}\right.
$$

Within the scope of the list of coloring algorithm, there are three kinds of algorithms: distributed greedy algorithm, distributed fair algorithm and distributed random algorithm. The three algorithms are defined according to the different goals. The actual cognitive radio is real-time network topology change, and color sensitive graph coloring algorithm is mainly used in the radio system of fixed topology. In network topology changes, need to recalculate network scheme, a huge communication overhead. Therefore, we revise the proposed methodologies into the following pattern.

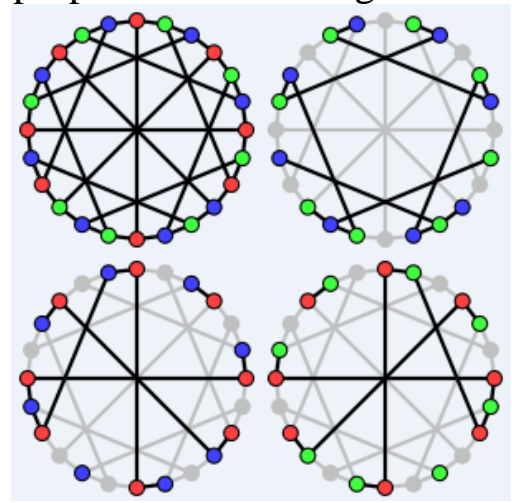

Figure 3. The Optimized and Enhanced Pattern for Graph Coloring

Assumes that the available frequency spectrum is divided into a series of basic spectral band are available, and also is the channel. Each channel bandwidth and transmission range is different, but the orthogonal to each other. Each node can detect locally available channel. Two users within a certain distance, that use the same channel at the same time can produce interference and the mathematical description of graph coloring model can by free matrix, the efficiency matrix, interference of matrix and matrix to represent the distribution. The enhanced objective function can be rewrited as follows.

$$
\arg \max \sum_{n=0}^{N-1} \log _{10}\left[\sum_{m=0}^{M-1} a_{n m} b_{n m}\right]
$$

In either spectrum allocation model, the spectrum allocation problem's main goal is to avoid the interference at the same time to maximize the basic utilization of the system, the channel capacity and fairness of give attention to two or more things, and reduce the communication cost, shorten the time. 


\section{Summary and Conclusion}

In this paper, we conduct research on the novel spectrum allocation algorithm based on the graph coloring. Network coding emphasizes the mutual cooperation between nodes, it from the perspective of network information theory, the application of the concept of this node cooperation coding to the whole communication network, so as to improve the overall performance of the network. At present, through the theoretical research and simulation implementation of the network coding, have shown the advantages of network coding in the network transmission, it not only can improve the network traffic, also in information reliability, security, etc., have great application prospect. With emergence of a variety of high performance low power devices and the improvement of location accuracy, more technology, the wireless channel multipath diversity combined technology and the development of the joint cross layer technology, nodes can have the corresponding signal processing ability which can implement complex algorithms, the future application of network coding will be more clear. In our approach, we combine the graphic coloring technique to form the optimized method that will enhance the performance of the traditional methodologies.

\section{Acknowledgements}

This research was financially supported by industrial science and technology research plan in Shaanxi Province of China (Program No. 2016GY-141).

\section{References}

[1] Barenboim, Leonid, and Michael Elkin. "Distributed Graph Coloring: Fundamentals and Recent Developments." Synthesis Lectures on Distributed Computing Theory 4.1 (2013): 1-171.

[2] Gebremedhin, Assefaw H., et al. "ColPack: Software for graph coloring and related problems in scientific computing." ACM Transactions on Mathematical Software (TOMS) 40.1 (2013): 1.

[3] Barenboim, Leonid, and Michael Elkin. "Combinatorial algorithms for distributed graph coloring." Distributed Computing 27.2 (2014): 79-93.

[4] Archetti, Claudia, Nicola Bianchessi, and Alain Hertz. "A branch-and-price algorithm for the robust graph coloring problem." Discrete Applied Mathematics 165 (2014): 49-59.

[5] Smith-Miles, Kate, et al. "Predicting metaheuristic performance on graph coloring problems using data mining." Hybrid Metaheuristics. Springer Berlin Heidelberg, 2013. 417-432.

[6] Xie, Chenning, et al. "Sync or async: Time to fuse for distributed graph-parallel computation." ACM SIGPLAN Notices. Vol. 50. No. 8. ACM, 2015.

[7] Djelloul, Halima, and Salim Chikhi. "Combining Bat Algorithm with angle modulation for Graph Coloring Problem." Symposium on Complex Systems and Intelligent Computing (CompSIC). 2015.

[8] Wang, Yuzhen, Chenghui Zhang, and Zhenbin Liu. "A matrix approach to graph maximum stable set and coloring problems with application to multi-agent systems." Automatica 48.7 (2012): 1227-1236. 